\title{
Relación entre mordida cruzada posterior unilateral y hábitos bucales deletéreos en preescolares de un municipio del sudoeste del Bahía
}

Alice Katarina Rosa Araújo Pina, ${ }^{1}$ Patricia Maria Coelho, ${ }^{2}$ Milena Tavares de Carvalho, ${ }^{3}$ Ana Flávia Soares, ${ }^{4}$ Leandro Morais Sousa. ${ }^{5}$

Resumen

Objetivo: El objetivo de esta investigación fue verificar la prevalencia de maloclusión en niños preescolares de un municipio del suroeste de Bahía, evaluando la presencia de mordida cruzada posterior unilateral y su relación con hábitos bucales deletéreos. Materiales y Métodos: La investigación fue realizada en niños matriculados en guarderías públicas y convenidas del municipio de Vitória da Conquista, BA, Brasil; después del cálculo muestral, la muestra incluyó 440 niños con edades de
3 a 5 años. La presencia de maloclusión fue evaluada clínicamente y un cuestionario para evaluar la relación con los hábitos fue respondido por los representantes. Resultados: Se encontró un porcentaje de $94,28 \%$ de los casos con mordida cruzada posterior unilateral relacionada a hábitos bucales. Conclusiones: Se hace evidente que los hábitos de succión no nutritiva pueden ser un factor etiológico para la mordida cruzada posterior unilateral.

Palabras clave: Mordida cruzada, hábitos, preescolares.

Artigo original

\section{Relação entre mordida cruzada posterior unilateral e hábitos bucais deletérios em pré-escolares de um município do sudoeste da Bahia}

Resumo

Objetivo: $O$ objetivo da pesquisa foi verificar a prevalência da má oclusão em crianças pré-escolares de um município do sudoeste baiano, enfocando a presença

\footnotetext{
${ }^{1}$ Graduación en Odontología. Faculdade Independente do Nordeste. Vitória da Conquista, Brasil.

2. Maestro en Ortodoncia. Pontifícia Universidade Católica de Minas Gerais, Belo Horizonte, Brasil

${ }^{3}$ Maestro en Ortodoncia. São Leopoldo Mandic. Campinas, Brasil.

4. Maestra y Doctora em Odontología. Universidade de São Paulo Campus Bauru. Bauru, Brasil.

${ }^{5}$ Cirujano Dentista. Faculdade Independente do Nordeste. Vitória da Conquista, Brasil.
} 
de mordida cruzada posterior unilateral e sua relação com hábitos bucais deletérios. Materiais e métodos: A pesquisa foi realizada em crianças matriculadas em crechespúblicaseconveniadasdomunicípio de Vitória da Conquista, BA, Brasil; após cálculo amostral, a amostra contemplou 440 crianças com idades entre os 3 e os 5 anos. A presença de má oclusão foi avaliada clinicamente e um critério para avaliar a relação com os hábitos foi respondido pelos responsáveis. Resultados: Encontrou-se uma porcentagem de $94,28 \%$ dos casos com mordida cruzada posterior unilateral relacionada aos hábitos bucais. Conclusão: Nota-se que os hábitos de sucção não nutritivo podem ser um fator etiológico para a mordida cruzada unilateral posterior.

Palavras chave: Mordida cruzada, hábitos, pré-escolares.

Original article

\section{Relationship between unilateral posterior crossbite and deleterious oral habits in preschool children from a municipality in southwestern Bahia.}

\begin{abstract}
Objective: The objective of the study was to verify the prevalence of malocclusion in pre-school children in a municipality in southwest Bahia, focusing on the presence of unilateral posterior crossbite and its relation with deleterious oral habits. Materials and methods: The research was carried out in children enrolled in public day care centers and in the city of Vitória da Conquista, BA, Brasil. After sample calculation, the sample consisted of 440
\end{abstract}

\section{Introducción}

La dentición participa activamente en la realización de tres funciones bucales, la fonación, la deglución y la masticación. Para el desarrollo correcto de estas funciones es necesario un trabajo equilibrado de la articulación temporal children aged 3 to 5 years. The presence of malocclusion was clinically evaluated and a questionnarie for evaluating the relationship with habits was answered by those represented. Results: We found that $94.28 \%$ of the cases with unilateral posterior crossbite were related to oral habits. Conclusions: It was found that nutritional sucking habits may be an etiological factor for posterior unilateral crossbite.

Key words: Cross bite, habits, pre-school.

mandibular, y engranaje perfecto entre los dientes, que cuando está ausente, además de comprometer las funciones desarrolladas, favorece la aparición de maloclusión. ${ }^{1-8}$

La maloclusión es el término usado cuando existen anormalidades en la 
dentición, presencia del apiñamiento, espaciamiento, giroversión, o cuando los dientes superiores se sobreponen a los dientes inferiores, del modo que las cúspides de los molares superiores no ocluyen con los surcos de los molares inferiores. La etiología de esta condición viene de factores hereditarios, congénitos, adquiridos del orden local o general y por la presencia de los hábitos bucales deletéreos; dietas pastosas, respiración bucal, onicofagia y el biberón también contribuyen. ${ }^{1-2}$

Los hábitos bucales deletéreos, incluyen succión digital, succión del chupón o chupete y onicofagia; son básicamente una repetición del acto que se extiende en el tiempo y que pueden tornarse resistente a los cambios. Los hábitos de succión no nutritiva, cuando son prolongados, intensos y frecuentes, pueden originar deformidades dentales y en la estructura facial, entre ellos: mordida abierta anterior, mordida cruzada posterior bilateral (MCPB) y mordida cruzada posterior unilateral (MCPU). ${ }^{3}$

La mordida cruzada posterior unilateral (MCPU) es la relación anormal vestíbulolingual en la oclusión, y causa alteración en la función de los $\operatorname{arcos}^{1,4-5}$ Es una maloclusión común. Cuando no se trata precozmente puede alterar la formación facial u ocasionar asimetría ósea en la cara. ${ }^{6-8}$ El paciente con mordida cruzada posterior unilateral, tiende a tener contacto prematuro en los caninos en relación céntrica (RC), ese contacto puede estimular al desplazamiento lateral de la mandíbula para poder encontrar una posición estable en máxima intercuspidación habitual $(\mathrm{MIH})$, pasando a tener una mordida cruzada posterior unilateral funcional. ${ }^{8}$
El presente estudio fue realizado con el objetivo de verificar la prevalencia de la maloclusión en niños preescolares de un municipio del suroeste de Bahía, enfocando la presencia de mordida cruzada posterior unilateral y su relación con hábitos bucales deletéreos.

\section{Materiales y Métodos}

El presente trabajo es un estudio transversal y los datos fueron sometidos y aprobados por el Comité de Ética en Investigación en Seres Humanos de la Facultad de Independente do Nordeste. (CAAE: 55463916.3.0000.5578).

El estudio fue realizado en niños de 3 a 5 años matriculados en guarderías públicas y convenidas del municipio de Vitória da Conquista, Bahia, Brasil. Este grupo etario fue elegido por la cronología de erupción dental, pues a los tres años de edad los dientes primarios se encuentran erupcionados y en oclusión y la erupción de los dientes permanentes se inicia alrededor de los cinco a seis años de edad. ${ }^{9}$ Para definir el número de la muestra, se realizó primeramente el cálculo muestral donde se contempló un nivel de confianza de $95 \%$, error alfa de $5 \%$ y $25 \%$ de posibilidades de pérdida de muestra. La muestra incluyó 440 niños, de un total de 3634 individuos, la selección fue realizada por muestreo aleatorio estratificado. Se adoptaron como criterios de inclusión: niños de ambos sexos y con edad establecida entre 3 a 5 años cuyos padres aceptaron la participación de estos en la investigación. Los criterios de exclusión fueron la ausencia de dientes 
anteriores, presencia de gran destrucción coronaria, niños sindrómicos y portadores de alteraciones patológicas.

El examen de la oclusión dental fue practicado por un único examinador debidamente calibrado en sala reservada en los locales de las instituciones. Se realizó con el auxilio de una espátula de madera desechable, con visión directa, bajo luz natural, como en el estudio de Massuia et al. ${ }^{10}$ Se consideraron las siguientes alteraciones oclusales: mordida abierta anterior, cuando el niño presentaba sobremordida negativa de cualquier magnitud; ${ }^{11}$ mordida cruzada posterior unilateral y bilateral, cuando las cúspides vestibulares de los dientes posteriores superiores ocluían en los surcos oclusales de los inferiores, ${ }^{12}$ y la mordida cruzada anterior, identificada cuando uno o más incisivos superiores ocluían en posición lingual con respecto a los incisivos inferiores. ${ }^{13}$ Cada niño fue llamado a la vez y ubicado de frente al examinador, de pie y con la cabeza levemente erguida hacia atrás.

Después de la realización del examen para identificar la presencia o no de la maloclusión, se efectuó una segunda evaluación que fue realizada por medio de un cuestionario autoaplicable enviado a los padres y/o responsables de los niños evaluados. El mismo fue compuesto inicialmente con la identificación, conteniendo el nombre del niño y el del responsable, la edad y nombre de la guardería en que estaba matriculado. En la segunda parte se se incluyeron tres interrogantes con respuestas excluyentes afirmatvas o negativas sobre succión digital, uso de biberón y chupete. Este levantamiento tuvo la finalidad de verificar la presencia de mordida cruzada posterior unilateral y su asociación con la presencia de los hábitos bucales deletéreos como principal etiología.

La edad y el género de los participantes se expresaron como frecuencias absoluta y relativa. Para evaluar la relación de la MCPU con la presencia de hábitos deletéreos se realizaron tablas de contingencias por separado: MCPU x Uso del Chupete; MCPU x Uso de biberón; MCPU x succión digital. Se utilizaron las pruebas Chi-cuadrado y Exacto de Fisher, analizadas por el programa BioEstat $5.3 \mathrm{y}$ nivel de significancia establecido en el 5\%.

\section{Resultados}

Los análisis de los resultados involucraron a 440 niños, de los cuales el 53\% fueron del sexo masculino y el $47 \%$ del sexo femenino. En cuanto a la edad, la distribucón resulto similar cada edad incluyendo 32,3\% los de 3 años, 35,0\% los de cuatro años y 32,7\% los de cinco años. De todos los niños evaluados, 94 (21,4\%) presentaron algún tipo de anormalidad oclusal, siendo halladas 113 (25,62\%) tipos de maloclusiones presentadas de forma exclusiva y/o asociadas a uno o más problemas oclusales.

La mordida cruzada posterior unilateral fue identificada en 35 (30,97\%) casos, se encontró asociada a la mordida abierta anterior en 11 casos. De estos 35 casos, relacionados con los hábitos bucales los hábitos bucales, 54,28 \% niños con MCPU refirieron hacer uso del biberón y 
chupete asociados, 5,7 \% uso del chupete y succión digital; el 5,7 \% también hizo uso del biberón y succión digital y el $22,8 \%$ niños con MCPU utilizaron solamente el biberón y 14,28 \% solamente el chupete. De los 35 niños con la mordida cruzada posterior unilateral presente, en 33 de los casos hubo asociación con hábitos bucales deletéreos.
Estos datos y otras asociaciones entre la MCPU se pueden ver en los gráficos 1 y 2 .

Con respecto a la distribución de la MCPU por género, se observó una mayor prevalencia en los pre-escolares del sexo femenino $(54,28 \%)$ que del masculino $(45,71 \%)$.

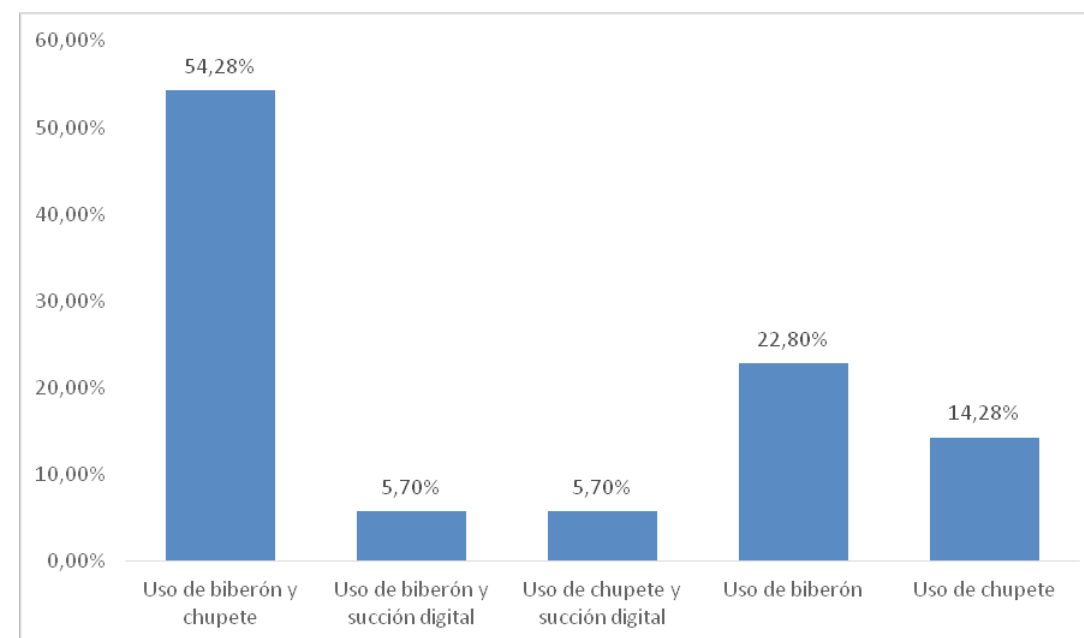

Gráfico 1: Prevalencia de hábitos deletéreos en niños con mordida cruzada posterior unilateral con uso del biberón, chupete y succión digital.

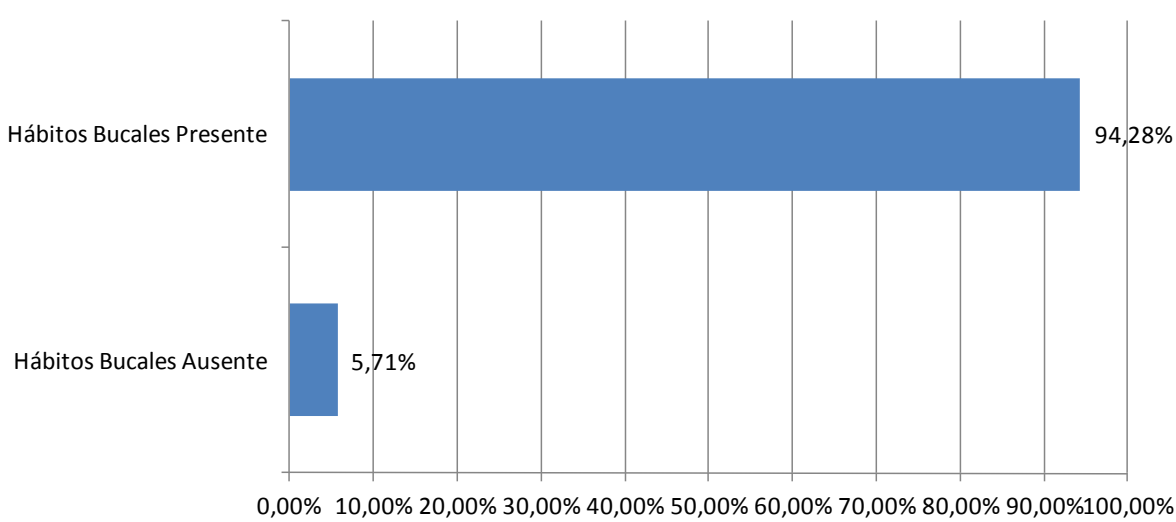

Gráfico 2: Asociación de la mordida cruzada posterior unilateral con hábitos bucales deletéreos $(p<0,05)$. 
De los niños con MCPU, el 40\% estuvo en el grupo de edad de tres años, el 22,85\% en el de cuatro años y el $37,14 \%$ en el de cinco años. (Tabla 1).

Tabla 1. Relación de la Mordida cruzada posterior unilateral según género y grupo de edad.

\begin{tabular}{|lcc|}
\hline Género & $(\mathrm{n})$ & $(\%)$ \\
\hline Femenino & 19 & $(54,28 \%)$ \\
Masculino & 16 & $(45,71 \%)$ \\
\hline Edad & & \\
3 años & 14 & $(40 \%)$ \\
4 años & 8 & $(22,85 \%)$ \\
5 años & 13 & $(37,14 \%)$ \\
\hline
\end{tabular}

$\mathrm{Al}$ evaluar la relación entre la MCPU y el uso de chupete, se encontró que entre los 66 individuos que hacen uso del mismo, la MCPU se hizo presente en $23(34,8 \%)$, mientras que de los 354 niños que no hacen uso del chupete sólo el 12 (3,4\%) presentaba la MCPU, observándose la relación directa entre el uso del chupete con el aumento de la prevalencia de esta maloclusión $(\mathrm{p}<0,05)$ (Tabla 2$)$.

La relación se puede observar también cuando el hábito deletéreo era el uso del biberón $(\mathrm{p}<0,05)$, pues sólo 6 niños de los

Tabla 2. Relación entre la presencia de MCPU y uso de chupete

\begin{tabular}{|c|c|c|c|}
\hline & $\begin{array}{l}\text { Presencia } \\
\text { de MCPU }\end{array}$ & $\begin{array}{c}\text { Ausencia de } \\
\text { MCPU }\end{array}$ & Valor de $\mathrm{p}^{*}$ \\
\hline $\begin{array}{l}\text { Presencia } \\
\text { de Chupete }\end{array}$ & 23 & 43 & \\
\hline & & & $<0,05$ \\
\hline $\begin{array}{l}\text { Ausencia } \\
\text { de Chupete }\end{array}$ & 12 & 342 & \\
\hline
\end{tabular}

*valor de p obtenido por test Chi-Cuadrado
360 que no usaron biberón presentaron la MCPU, la maloclusión fue reportada en 29 los individuos de un total de 80 que si hicieron uso del biberón (Tabla 3).

Tabla 3. Relación entre la presencia de MCPU y el uso de biberón

\begin{tabular}{|c|c|c|c|}
\hline & $\begin{array}{l}\text { Presencia } \\
\text { de MCPU }\end{array}$ & $\begin{array}{c}\text { Ausencia de } \\
\text { MCPU }\end{array}$ & Valor de $\mathrm{p}^{*}$ \\
\hline $\begin{array}{l}\text { Presencia } \\
\text { de biberón }\end{array}$ & 29 & 51 & \\
\hline & & & $<0,05$ \\
\hline $\begin{array}{l}\text { Ausencia } \\
\text { de biberón }\end{array}$ & 6 & 354 & \\
\hline
\end{tabular}

*valor de p obtenido por el test de Chi-Cuadrado

En cuanto a la succión digital, a pesar de no ser un hábito deletéreo presente en muchos niños, hizo que la prevalencia de la MPCU aumentara más de 4 veces cuando era comparada en los niños que no poseían el hábito $(\mathrm{p}<0,05)$. De los 13 individuos que hacen succión digital, 4 presentaban la MCPU (30,8\%), mientras que en el grupo que no posee ese hábito deletéreo apenas $7,3 \%$ (31) de los niños presentaron la maloclusión (Tabla 4).

Tabla 4. Relación entre la presencia de MCPU y el uso de biberón

\begin{tabular}{|lccc|}
\hline & $\begin{array}{c}\text { Presencia } \\
\text { de MCPU }\end{array}$ & $\begin{array}{c}\text { Ausencia } \\
\text { de MCPU }\end{array}$ & $\begin{array}{c}\text { Valor } \\
\text { de }{ }^{*}\end{array}$ \\
\hline $\begin{array}{l}\text { Presencia de } \\
\text { Succión digital }\end{array}$ & 4 & 9 & \\
\hline & & & $<0,05$ \\
\hline $\begin{array}{l}\text { Ausencia de } \\
\text { Succión digital }\end{array}$ & 31 & 396 & \\
\hline
\end{tabular}

*valor de p obtenido por el test Exacto de Fisher 


\section{Discusión}

Actualmente la prevalencia de la maloclusión en preescolares ha sido ampliamente estudiada, debido a que puede afectar las funciones normales, llegando a alterar el sistema estomatognático, lo cual se considera un problema de salud pública en el mundo.

En el presente estudio, se observaron los tipos de maloclusión en preescolares de un municipio del suroeste de Bahía, relacionándolos con género, edad $\mathrm{y}$ hábitos bucales deletéreos. ${ }^{9,23,25,26,27}$

En este estudio se encontraron 113 casos de maloclusión, observados en una población del 440 niños de 3 a 5 años, con una prevalencia de $21,4 \%$. Otras investigaciones reportaron hallazgos similares al evaular la prevalencia de las maloclusiones. Scarpelli e Cândido et al. hallaron una prevalencia del 53,3\% y del 43,6\% respectivamente. En 2010 se realizó un estudio en el suroeste de Bahía, Brasil donde se reveló un porcentaje de maloclusión del $66,7 \%$ y en la región Nordeste del 64,8\%, evidenciándose resultados superiores a los encontrados en la población de este estudio. ${ }^{29-30}$

A pesar de que no se considera una diferencia significativa según la literatura, los resultados de esta investigación se diferencian de los estudios de Rossi et al, quienes reportaron el 56\% se presentó en el sexo masculino, mientras que el sexo femenino tuvo un índice del $44 \%$. ${ }^{2,31}$

Se reveló en el presente estudio que de todas las maloclusiones observadas, la prevalencia fue del 35\% para MCPU. Esta prevalencia es superior al comparar con los estudios realizados por Lopes y Emmerich en el municipio del Vitória (ES), en donde se obtuvo una prevalencia promedio del $8 \%$ al $16 \%$. A pesar del ser un problema común, actualmente el promedio de casos presentes tiene un bajo índice relativo a la mordida abierta anterior que es la primera maloclusión más encontrada en las investigaciones realizadas. Los niños portadores de mordida abierta anterior junto a la mordida cruzada posterior unilateral representaron el $11,7 \%$, indicando que las anomalías no tienen relación con el surgimiento mutuo. ${ }^{1,10,25,28}$.

Moyers clasificó la MCPU, según su etiología, en: odontológica, ósea y muscular. A pesar de la diversidad de factores relacionados con la etiología de esa maloclusión, los hábitos orales deletéreos están íntimamente ligados a estos, ${ }^{10,24}$ factor que puede observarse en el estudio en cuestión.

Los hábitos bucales deletéreos son considerados factores etiológicos relevantesenlaaparición de maloclusiones. En el presente estudio se observó que 94,28\% de los niños con MCPU presentó hábitos deletéreos.

Hallazgos similares fueron descritos por descrita por Thomaz y Valencia quienes revelaron un índice de $72,1 \%$; similar también al resultado presentado por Emmerich et al que fue del 82,2\%.

Evidencias científicas revelan que cuando los hábitos de biberón y chupete persisten, pueden alterar el sistema estomatognático. ${ }^{11,19}$ Esto está en concordancia con lo observado en la presente investigación en la cual la prevalencia del uso del biberón y el chupete fue de $22,8 \%$ y $14,28 \%$ respectivamente. 
Después de la confirmación del Conclusión diagnóstico, es imprescindible la planificación eficiente de la corrección, independientemente de la etapa del desarrollo, para evitar tanto problemas oclusales en el adulto, como asimetría facial. ${ }^{18-24,28}$ El profesional debe enfocarse en la masticación correcta, adecuando un desarrollo morfológico y funcional, permitiendo que el sistema estomatognático desempeñe su función y principalmente que devuelva la oclusión normal del paciente. ${ }^{9,18-19,23}$
Enelpresenteestudio, fueposibleverificar que la prevalencia de la maloclusión en niños preescolares de un municipio del suroeste de Bahía es del $21,4 \%$, ese índice está directamente relacionado con los hábitos bucales deletéreos prolongados, pues el uso del chupete, biberón y la succión digital aumentó de forma significativa la presencia de la mordida cruzada posterior unilateral.

\section{Referencias bibliográficas}

1. Wagner $Y$, Weltzien HR. Occlusal characteristics in 3-year-old children - results of a birth cohort study. BMC Oral Health. 2015; 15: 1-6.

2. Leôncio LL, Furtado AFKK, Chacon DL, Nóbrega CBC, Costa DEL, Queiroz SF. Prevalência de máoclusão em crianças de cinco anos de idade do município de Patos, PB. Arq Odontol. 2015; 51: 25-31.

3. Silva LE. Hábitos bucais deletérios. RPM. 2006; 20: 47-50.

4. Pacheco BA, Silva TMA, Mazzamo LC, Berwing CL, Neu PA; Relação da respiração oral e hábitos de sucção não nutritiva com alterações do sistema estomatognático. CEFAC. 2012; 14: 206-210.

5. Trannin GP, Maffei C, Alanis ARL, Camargo SE, Lara VSM. Características da função mastigatória em indivíduos com mordida cruzada posterior unilateral. Arch Oral Res. 2012; 8: 127-132.

6. Avila DRBN, Novo FN, Juliano Y, Marinez AJ, Júnior CV. Estudo dos sinas de escoliose em crianças com ou sem mordida cruzada posterior unilateral. Rev Bras Ciên Saúde. 2010; 8: 27-34.

7. Schiavinato J, Vedodello SAS, Valdrigui H, Vedovello Filho M, Lucato SA. Assimetria facial em indivíduos com mordida cruzada posterior por meio de fotografias. RGO. 2010; 58:81-83.

8. Silva Filho GO, Silva BRP, Rego NNVM, Capelozza Filho L. Epidemiologia da mordida cruzada posterior na dentadura decídua. J Bras Odontopediatr Odontol bebê. 2003; 6: 61-68.

9. Massuia MJ, Carvalho OW, Matsuo T. Má oclusão, hábitos bucais e aleitamento materno: Estudo de base populacional em um município de pequeno porte. PBOCI. 2011; 11:451-457.

10. Locks A, Weissheimer A, Ritter ED, Ribeiro ULG, Menezes ML, Derech ADC, et al. Mordida cruzada posterior: uma classificação mais didática. R Dental Press Ortodon Ortop Facial. 2008; 13:146-158.

11. Matos CG, Santos CJ, Granzotti GBR, Silva K, Baldrighi MZES, César RAHPC. A prevalência de hábitos orais em pré-escolares. Distúrb Comun. 2017; 29: 68-76.

12. Carvalho CM, Carvalho LFP, Forte FDS, Aragão MS, Costa LJ. Prevalência de mordida aberta anterior em crianças de 3 a 5 anos em Cabedelo/PB e relação com hábitos bucais deletérios. Pesqui bras odontopediatria clín Integr. 2009; 9: 205-220.

13. Johanns CM, Silvério K, Furkim AM, Marchesan I. Há relação de hábitos orais deletérios com a tipologia facial e a oclusão dentária. Rev CEFAC. 2011; 13: 1-8.

14. Zollner MSAC, Jorge AOC. Aleitamento materno: caminho natural para a saúde. J Bras Odontopediatr Odontol Bebê. 2005; 8: 135-142.

15. Freire GML, Cárdenas ABC, Deza JEES, Torrent JMU, Oliveira LB, Quesada JRB. Exploring the association 
between feeding habits, non-nutritive sucking habits, and malocclusions in the deciduous dentition. Prog Orthod. 2015; 16:43.

16. Scavone Junior H, Ferreira RI, Mendes TE, Ferreira FV. Prevalence of posterior crossbite among pacifier users: a study in the deciduous dentition. Braz Oral Res. 2007; 21(2): 153-161.

17. Osorio BIDDC, Rodrigues SAMCM, Pruneda MFJ, Nuñes MMV. Prevalencia y factores de riesgo de mordida cruzada posterior en niños de 4-9 años de edad en ciudad Nezahualcóyotl. Bol Med Hosp Infant Mex. 2004; 61(2): 141- 148.

18. Cuoghi AO, Mendonça RM, Zamalloa MMY, Mordida cruzada posterior. Corrección y consideraciones. Caso clínico con 7 años de seguimento. Acta Odontol Venez 2011; 49: 1-9.

19. Nova VF, Castillo QAJ, Treviño OS, González SHA, Nuncio SRH, Guzmán BJF, et al. Association between allergic rhinitis, bottle feeding, non-nutritive sucking habits, and malocclusion in the primary dentition. Arch Dis Child. 2006; 91: 836-840.

20. Van Der Linden FPGM. A dentição decídua. In: Van Der Linden FPGM. Ortodontia: desenvolvimento da dentição. São Paulo: Quintessence; 1986. P.29-31.

21. Moyers RE. Classificação e terminologia da má-oclusão. In: Moyers RE. Ortodontia. Rio de Janeiro: Guanabara Koogan; 1991. P.156-157.

22. Rossi LB, Pizzol KEDC, Boeck EM, Lunardi N, Garbin AJI. Correção de mordida cruzada anterior funcional com a terapia de pistas diretas planas: relato de caso. Fac Odont Lins/Unimep. 2012; 22(2): 45-50.

23. Espinosa QTM, Brito MI, Interferencias oclusales y su relación con las maloclusiones funcionales en niños con dentición mixta. Rev méd electrón. 2010; 32(2).

24. Peiro CA. Interceptive orthodontics: The need for early diagnosis and treatment of posterior crossbites. Med Oral Patol Oral Cir Bucal. 2006; 11: 210-4.

25. Mata J, Zambrano F, Quirós O, Maza P, D'Jurisic A, Alcedo C, Fuenmavor D, Ortiz M. Expansión rápida de maxilar en maloclusiones transversales: revisión bibliográfica. Rev Latinoam Odontoped Orto. 2009; 24: 1-27.

26. Silva Filho OG, Santamaria Jr MM, Capelozza Filho L. Epidemiology of Posterior Crossbite in the Primary Dentition. J Clin Pediatr Dent. 2007; 32: 73-8.

27. Moimaz SAS, Lolli FL, Garbin SAC, Saliba O, Saliba AN, Azevedo SP. Harmful oral suction habits in children: association with breastfeeding and family social profile. Rev odonto ciênc. 2010; 25: 355-60.

28. Malandris M, Mahoney EK. Aetiology, diagnosis and treatment of posterior cross-bites in the primary dentition. Intl J Paed Dent. 2004; 14: 155-66.

29. Scarpelli AC, Paiva SM, Viegas CM, Carvalho AC, Ferreira FM, Pordeus IA. Oral healthrelated quality of life among Brazilian preschool children. Community Dent Oral Epidemiol. 2013; 41: 336-44.

30. Cândido IRF, Figueiredo ACP, Cysne SS, Santiago BM, Valença AMG. Características da oclusão decídua em crianças de 2 a 5 anos em João Pessoa -PB-Brasil. Pesq Bras Odontoped Clin Integr. 2010; 10: 15-22.

31. Alves JAO, Forte FDS, Sampaio FC. Condição socioeconômica e prevalência de más oclusões em crianças de 5 e 12 anos na USF Castelo Branco III: João Pessoa/Paraíba. Rev Dent. Press Ortodon Ortopedi Facial. 2009; 14: 52-59.

Recibido: $13 / 04 / 18$

Aceptado: 06/07/18

Correspondencia: Alice Katarina Pina alicearaujopina@hotmail.com 